\title{
Uplink Multiuser MIMO-OFDM System in the Presence of Phase Noises, Power Imbalance, and Correlation
}

\author{
Xiaoming Chen $\mathbb{D},{ }^{1}$ Andreas Wolfgang, ${ }^{2}$ and Tommy Svensson $\mathbb{D D}^{3}$ \\ ${ }^{1}$ School of Electronic and Information Engineering, Xian Jiaotong University, Xi'an 710049, China \\ ${ }^{2}$ Qamcom Research \& Technology AB, 41285 Gothenburg, Sweden \\ ${ }^{3}$ Department of Electrical Engineering, Chalmers University of Technology, 41296 Gothenburg, Sweden
}

Correspondence should be addressed to Xiaoming Chen; xiaoming.chen@mail.xjtu.edu.cn

Received 3 December 2017; Accepted 14 January 2018; Published 8 February 2018

Academic Editor: Ting-Yen Shih

Copyright (C) 2018 Xiaoming Chen et al. This is an open access article distributed under the Creative Commons Attribution License, which permits unrestricted use, distribution, and reproduction in any medium, provided the original work is properly cited.

\begin{abstract}
The effects of phase noises (PNs), power imbalances, and correlations on multiuser orthogonal frequency division multiplexing (OFDM) multiple-input multiple-output (MIMO) systems are studied. It is assumed that each user is equipped with a single antenna, whereas the base station (BS) has multiple antennas and use zero-forcing (ZF) decoder for multiuser detection. Since each user has an independent oscillator, the received uplink (UL) signal at each BS antenna is corrupted by all of these independent PNs. Furthermore, there may be power imbalances and correlations (due to common scatterers) between different users. These impairments are jointly analyzed in this work. A closed-form expression of the mean square error (MSE) performance of the multiuser MIMO-OFDM system is derived. The analytical results are verified by simulations.
\end{abstract}

\section{Introduction}

The orthogonal frequency division multiplexing (OFDM) technique [1] can effectively turn a frequency-selective channel [2] into multiple frequency-flat subchannels at different subcarriers, allowing the simple one-tap channel equalization to mitigate the multipath effect. Recently, it is chosen as the main fifth generation (5G) waveform for sub-6 GHz as well as millimeter-wave bands $[3,4]$ by the 3 rd generation partnership project (3GPP) standardization [5]. The OFDM can be readily combined with multiple-input multiple-output (MIMO) techniques [6-8] to exploit spatial multiplexing and/or diversity gain. As a result, MIMO-OFDM systems are ubiquitously employed in modern telecommunications, such as long-term evolution (LTE) and wireless local area network (WLAN) systems, for example, $[9,10]$.

Unfortunately, MIMO-OFDM systems are sensitive to oscillator phase noises [11-23], which cause common phase error (CPE) and intercarrier interference (ICI). While the $\mathrm{CPE}$ is a common phase rotation for all the subcarriers (of one OFDM symbol), the ICI represents the interferences caused by nonorthogonal subcarriers. The PN effect on single-user MIMO-OFDM (SU-MIMO-OFDM) systems has been well studied in the literature [11-19]. Most of the works assume a common oscillator for all the antennas at the transceiver [1116], whereas the case of an independent oscillator for each antenna has been studied in [17-19]. Assuming the base station (BS) antennas share a common oscillator, the downlink (DL) of the multiuser MIMO-OFDM (MU-MIMO-OFDM) system (at each user) is similar to the single-user case (in that the user see only a single transmit PN). In the uplink (UL) transmission, however, the received signal at the BS is corrupted by multiple transmit PNs. Since the PNs from different users are independent, the PN estimation in the UL is more complex than that in the DL. For this reason, we focus on the UL multiuser PN effect in this work.

There are a few works studying the PN effect on uplink MU-MIMO-OFDM systems, for example, [20, 21]. While [20] considered multiple PNs from the users together with another PN at the BS, [21] considered multiple PNs at the BS (with large antenna array) with PN-free users. The PNs from multiple users are mixed at each BS antenna, whereas the PNs (of multiple oscillators) at the BS are uncoupled. Therefore, $\mathrm{PN}$ estimation in the former is more challenging than that in the latter. For example, the CPEs of multiuser PNs must 
be jointly estimated [20], whereas the CPE of each of the BS PNs can be tracked separately in an easier way $[21,22]$. It is difficult to compensate the ICI effects of multiple transmit PNs at the receiver. As a result, a PN compensation scheme that mitigates the multiple transmit PNs and a receive PN separately was proposed in [23] for multinode backhauling. Nevertheless, the scheme requires a feedback loop with direct $\mathrm{RF}$ sampling at each transmitting node and, therefore, is not feasible for the considered multiuser scenario in this work.

In this work, we focus on the ICI effects of multiuser PNs. Specifically, we derive a closed-form expression of the mean square error (MSE) performance of the uplink MUMIMO-OFDM system with and without power imbalance and correlation, assuming the CPEs of different PNs can be perfectly estimated. (Given the fact that the $\mathrm{CPE}$ is just the time average of the PN over one OFDM symbol, the CPE can usually be estimated accurately [11, 14, 15]. The effect of imperfect CPE estimation is included in simulations.) Unlike [20] where the multiuser PN effects were studied by simulation solely, the analytical results derived in this work give more insight into the ICI effect of multiuser PNs. Moreover, the derived MSE expression can take into account of power imbalances and correlations between different users. (In the literature, it is usually assumed that spatially separated users are uncorrelated. Nevertheless, measurements and simulations confirm that spatially separated users can still have correlations due to common scatterers [24-26].) To the best knowledge of the authors, the joint effects of multiuser PNs, power imbalance, and correlation of the uplink MU-MIMO-OFDM system have not been studied analytically in the previous literature. Finally, the ICI effect of multiuser PNs for different numbers of BS antennas and users are studied analytically and experimentally (by simulations). Good agreements between simulated and theoretical MSEs are observed.

Notations 1. Throughout this paper, boldface lower and uppercase letters (e.g., $\mathbf{x}$ and $\mathbf{X}$ ) represent column vector and matrix, respectively; $\operatorname{diag}(\mathbf{x})$ denotes a diagonal matrix whose diagonal elements are given by $\mathbf{x} ;\|\mathbf{x}\|$ is the Euclidean norm of $\mathbf{x} ; \operatorname{Tr}(\mathbf{X})$ represents the trace of $\mathbf{X} ; \mathbf{I}_{M}$ is an $M \times$ $M$ identity matrix; $j=\sqrt{-1} ; \otimes$ denotes the Kronecker product; $E$ represents mathematical expectation; and $T, H$, and $\dagger$ denote transpose, transpose conjugate (Hermitian), and Moore-Penrose pseudoinverse, respectively.

\section{System Model}

For simplicity, we assume $K$ users (each equipped with a single antenna along with a free-running oscillator) and $M(>$ $K$ ) antennas at the BS (see Figure 1). The BS uses zero-forcing (ZF) decoder for multiuser detection. Throughout this paper, we assume that the cyclic prefix (CP) of the OFDM symbol is longer than the channel spread.

Let $N$ be the number of OFDM subcarriers and $\mathbf{F}$ be an $N \times N$ unitary discrete Fourier transform (DFT) matrix, whose elements are given by $\exp (-j 2 \pi n l / N) / \sqrt{N},(n, l=$ $0, \ldots, N-1)$, and $\varphi_{k}$ and $\boldsymbol{\theta}$ be $N \times 1$ vectors consisting of

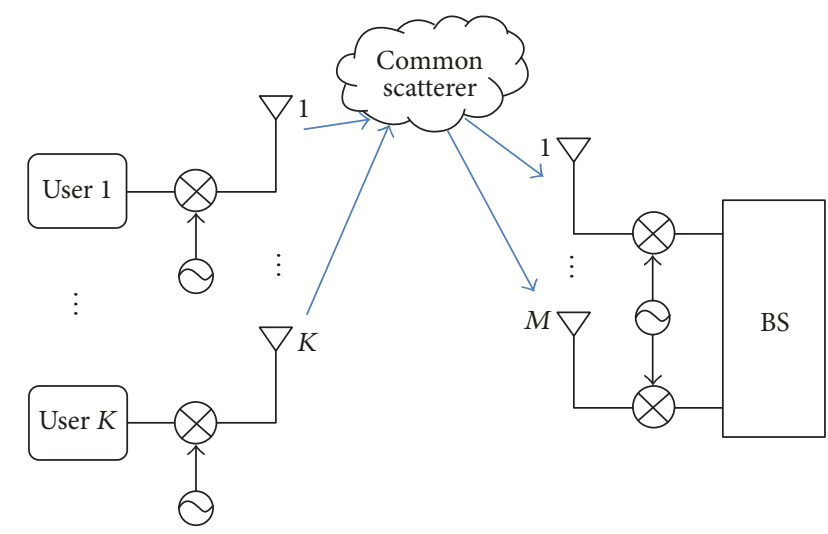

FIGURE 1: Block diagram of the considered MU-MIMO-OFDM system with illustrations of oscillators and a common scatterer.

the time-domain PNs within one OFDM symbol at the $k$ th user $(k=1, \ldots, K)$ and at the BS, respectively. The frequencydomain expression of the UL transmission (from $K$ users to the BS) in the presence of PNs is given as follows [17]:

$$
\mathbf{y}=\left(\mathbf{G}_{\mathrm{R}} \otimes \mathbf{I}_{M}\right) \mathbf{H G}_{\mathrm{T}} \mathbf{x}+\mathbf{w},
$$

where $\mathbf{H}$ is an $M N \times K N$ block diagonal channel matrix whose $n$th diagonal block entry $\mathbf{H}_{n}$ is an $M \times K$ channel transfer function (CTF) matrix at the $n$th subcarrier, $\mathbf{x}=$ $\left[\begin{array}{llll}\mathbf{x}_{1}^{T} & \mathbf{x}_{2}^{T} & \cdots & \mathbf{x}_{N}^{T}\end{array}\right]^{T}$ is the $K N \times 1$ signal vector with $\mathbf{x}_{n}$ denoting the $K \times 1$ transmitted signal vector at the $n$th subcarrier, $\mathbf{y}=\left[\begin{array}{llll}\mathbf{y}_{1}^{T} & \mathbf{y}_{2}^{T} & \cdots & \mathbf{y}_{N}^{T}\end{array}\right]^{T}$ is the $M N \times 1$ signal vector with $\mathbf{y}_{n}$ denoting the $M \times 1$ received signal vector at the $n$th subcarrier, $\mathbf{w}$ is an $M N \times 1$ additive white Gaussian noise (AWGN) vector, $\mathbf{G}_{\mathrm{R}}=\mathbf{F} \operatorname{diag}(\exp (j \boldsymbol{\theta})) \mathbf{F}^{H}$ is an $N \times N$ matrix of the PN spectral components of the BS oscillator, and $\mathbf{G}_{\mathrm{T}}$ is a $K N \times K N$ matrix consisting of the $N$ spectral components of the PNs of all the $K$ users. The $(n, l)$ th entry of $\mathbf{G}_{\mathrm{R}}$ is denoted as $g_{(n-l)_{\mathrm{N}}}^{\mathrm{Rx}}$, where $(n-l)_{N}$ denotes $(n-l) \bmod N$. The $(n, l)$ th block of $\mathbf{G}_{\mathrm{T}}$ is $\operatorname{diag}\left(\mathbf{g}_{(n-l)_{N}}^{\mathrm{Tx}}\right)$, where the $K \times 1$ vector $\mathbf{g}_{(n-l)_{N}}^{\mathrm{Tx}}$ consists of the corresponding spectral components of all the PNs from the $K$ users. Note that (1) only holds approximately since the PN in the $\mathrm{CP}$ and the end of the time-domain OFDM symbol are different. Nevertheless, it is a good approximation [11, 15, 17]. By separating the CPE and ICI terms $\mathbf{G}_{\mathrm{T}}=\mathbf{I}_{N} \otimes \operatorname{diag}\left(\mathbf{g}_{0}^{\mathrm{Tx}}\right)+\mathbf{P}_{\mathrm{T}}$ and $\mathbf{G}_{\mathrm{R}}=g_{0}^{\mathrm{Rx}} \mathbf{I}_{N}+\mathbf{P}_{\mathrm{R}}$, (1) can be rewritten as

$$
\mathbf{y}=g_{0}^{\mathrm{Rx}} \mathbf{H}\left(\mathbf{I}_{N} \otimes \operatorname{diag}\left(\mathbf{g}_{0}^{\mathrm{Tx}}\right)\right) \mathbf{x}+\mathbf{e}+\mathbf{w},
$$

where $g_{0}^{\mathrm{Rx}}$ is the CPE of the PN at the BS, $\mathrm{g}_{0}^{\mathrm{Tx}}$ consists of the CPEs of the PNs from the $K$ users, and the ICI term $\mathbf{e}$ is

$$
\begin{aligned}
\mathbf{e}= & \left(\mathbf{P}_{\mathrm{R}} \otimes \mathbf{I}_{M}\right) \mathbf{H} \mathbf{P}_{\mathrm{T}} \mathbf{x}+g_{0}^{\mathrm{Rx}} \mathbf{H} \mathbf{P}_{\mathrm{T}} \mathbf{x} \\
& +\left(\mathbf{P}_{\mathrm{R}} \otimes \mathbf{I}_{M}\right) \mathbf{H}\left(\mathbf{I}_{N} \otimes \operatorname{diag}\left(\mathbf{g}_{0}^{\mathrm{Tx}}\right)\right) \mathbf{x} .
\end{aligned}
$$

The received signal at the $n$th subcarrier can be expressed as

$$
\mathbf{y}_{n}=\mathbf{H}_{n} \operatorname{diag}\left(\mathbf{g}_{0}\right) \mathbf{x}_{n}+\mathbf{e}_{n}+\mathbf{w}_{n}
$$


where $\mathbf{g}_{0}=g_{0}^{\mathrm{Rx}} \mathbf{g}_{0}^{\mathrm{Tx}}, \mathbf{e}_{n}$ and $\mathbf{w}_{n}$ denote ICIs and AWGNs at the $n$th subcarrier, respectively. The CPEs $\mathbf{g}_{0}$ of multiple PNs can be jointly estimated as follows [20]:

$$
\widehat{\mathbf{g}}_{0}=\frac{1}{N_{\mathrm{p}}} \sum_{n \in S_{\mathrm{p}}}\left(\mathbf{H}_{n} \operatorname{diag}\left(\mathbf{x}_{n}\right)\right)^{\dagger} \mathbf{y}_{n}
$$

where $S_{\mathrm{p}}$ denotes the set of the $N_{\mathrm{p}}$ scattered pilots. The CPEs can be corrected at the $\mathrm{ZF}$ decoder as

$$
\widehat{\mathbf{x}}_{n}=\operatorname{diag}\left(\widehat{\mathbf{g}}_{0}\right)^{-1} \mathbf{H}_{n}^{\dagger} \mathbf{y}_{n}
$$

where $\widehat{\mathbf{x}}_{n}$ denotes the detected signals at the $n$th subcarrier. Note that, in order to focus on the PN effect, we assume perfect channel estimation. In practice, the estimated channel in the preamble will contain an initial CPE. In the payload, the CPE will be different. Thus, it is necessary to estimate the relative $\mathrm{CPE}$ with respect to the initial CPE. Mathematically, it is equivalent to assume that the estimated channel contains the preamble CPE and then the relative CPE in the payload can still be estimated using (5).

For simplicity, we assume the Wiener PN model for the free-running oscillator, whose discrete-time expression is given as follows [11, 13-15]:

$$
\phi(i+1)=\phi(i)+\zeta(i)
$$

where $\phi$ is the PN and $\zeta$ is a zero-mean Gaussian random variable, whose variance is $4 \pi \beta T_{\mathrm{s}}$ with $T_{\mathrm{s}}$ denoting the sampling duration and $\beta$ representing the $3 \mathrm{~dB}$ bandwidth of the PN. The $3 \mathrm{~dB}$ bandwidth is related to the carrier frequency $\mathrm{fc}$ as $\beta=\varsigma \pi f_{c}^{2}$, where $\varsigma$ is a parameter characterizing the quality of the oscillator [27]. Nevertheless, the value of $\beta$ is usually given instead of $\varsigma$ in the literature.

In order to include power imbalances and correlations of different users, the MIMO channel impulse response of the $l$ th tap is modeled as $\widetilde{\mathbf{H}}_{l}=\mathbf{H}_{w} \mathbf{R}^{1 / 2}, \mathbf{H}_{w}$ denotes the spatially white MIMO channel with independent and identically distributed (i.i.d.) complex Gaussian variables, and $\mathbf{R}^{1 / 2}$ is the Hermitian square root of the correlation matrix $\mathbf{R}$, defined as

$$
\mathbf{R}=\operatorname{diag}(\sqrt{\boldsymbol{\eta}}) \boldsymbol{\Phi} \operatorname{diag}(\sqrt{\boldsymbol{\eta}})
$$

where $\boldsymbol{\eta}$ is a vector consisting of the mean effective gains (MEGs) [28] of the users' antennas, $\sqrt{ }$ represents elementwise square root, and the matrix $\Phi$ consists of correlation coefficients due to the common scatters. The power imbalances are the MEG differences (in $\mathrm{dB}$ ) between the elements in $\boldsymbol{\eta}$. It is noted that the path loss differences between different users can be included in the power imbalances.

\section{Performance Analysis}

In order to focus on the ICI effects of multiuser PNs, we assume perfect estimation of CPEs. Since the BS can afford high quality oscillator with negligible $\mathrm{PN}$ whereas the users are usually equipped with low-cost oscillators with nonnegligible PNs, we ignore the $\mathrm{BS} \mathrm{PN}$ and focus on multiuser PNs. (The BS PN and imperfect CPE estimation are considered in the simulations in the next section.) For notational convenience, we drop the superscript Tx hereafter.

The signal at the $n$th subcarrier after the $\mathrm{ZF}$ decoder is given as

$$
\mathbf{r}_{k}=\operatorname{diag}\left(\mathbf{g}_{0}\right) \mathbf{x}_{n}+\sum_{l=0, l \neq n}^{N-1} \operatorname{diag}\left(\mathbf{g}_{(n-l)_{N}}\right) \mathbf{x}_{l}+\mathbf{H}_{n}^{\dagger} \mathbf{w}_{n} .
$$

The (normalized) MSE at the $n$th subcarrier is

$$
\xi_{n}=\frac{E\left[\left\|\sum_{l=0, l \neq n}^{N-1} \operatorname{diag}\left(\mathbf{g}_{(n-l)}\right) \mathbf{x}_{l}\right\|^{2}\right]+E\left[\left\|\mathbf{H}_{n}^{\dagger} \mathbf{w}_{n}\right\|^{2}\right]}{E\left[\left\|\operatorname{diag}\left(\mathbf{g}_{0}\right) \mathbf{x}_{n}\right\|^{2}\right]} .
$$

Note that the normalized MSE is also referred to as error vector magnitude (EVM) [15], which is a popular performance metric in the industry.

Assuming the subcarrier symbols are independent and identically distributed with a variance of $\sigma_{x}^{2}$, that is, $E\left[\mathbf{x}_{n} \mathbf{x}_{n}^{H}\right]=\sigma_{x}^{2} \mathbf{I}_{K}$, the power contributions of the useful signal (with known CPEs) and ICI term can be in expressed as

$$
\begin{aligned}
E\left[\left\|\operatorname{diag}\left(\mathbf{g}_{0}\right) \mathbf{x}_{n}\right\|^{2}\right] & =\sigma_{x}^{2} E\left[\left\|\mathbf{g}_{0}\right\|^{2}\right], \\
E\left[\left\|\sum_{l \neq n} \operatorname{diag}\left(\mathbf{g}_{(n-l)_{N}}\right) \mathbf{x}_{l}\right\|^{2}\right] & =\sigma_{x}^{2} E\left[\sum_{l \neq n}\left\|\mathbf{g}_{(n-l)_{N}}\right\|^{2}\right],
\end{aligned}
$$

respectively.

Assuming the oscillators at different users are of the same quality (i.e., PNs from different users have the same $3 \mathrm{~dB}$ bandwidth $\beta$, the same CPE term $\sigma_{\mathrm{CPE}}^{2}=E\left[\left|g_{0}\right|^{2}\right]$, and the same ICI term $\left.\sigma_{\text {ICI }}^{2}=E\left[\sum_{l \neq n}\left|g_{(n-l)_{N}}\right|^{2}\right]\right)$,

$$
\begin{gathered}
E\left[\left\|\mathbf{g}_{0}\right\|^{2}\right]=K E\left[\left|g_{0}\right|^{2}\right]=K \sigma_{\mathrm{CPE}}^{2} \\
E\left[\sum_{l \neq n}\left\|\mathbf{g}_{(n-l)_{N}}\right\|^{2}\right]=K E\left[\sum_{l \neq n}\left|g_{(n-l)_{N}}\right|^{2}\right]=K \sigma_{\mathrm{ICI}}^{2}
\end{gathered}
$$

Note that $E\left[\sum_{l \neq n}\left|g_{(n-l)_{N}}\right|^{2}\right]=E\left[\sum_{n \neq 0}\left|g_{n}\right|^{2}\right]$. Let

$$
\left.g_{(n-l)_{N}}\right|_{l=0}=g_{n}=\frac{1}{N} \sum_{i} \exp (j \phi(i)) \exp \left(-\frac{j 2 \pi n i}{N}\right),
$$

and the total power of the $\mathrm{PN}$ is given as

$$
\begin{aligned}
\sum_{n=0}^{N-1}\left|g_{n}\right|^{2}= & \frac{1}{N^{2}} \sum_{n=0}^{N-1} \sum_{i} \sum_{p} \exp (j(\phi(i)-\phi(p))) \\
& \times \exp \left(j \frac{2 \pi k(p-i)}{N}\right) \\
= & \frac{1}{N^{2}} \sum_{i} \sum_{p} \exp (j(\phi(i)-\phi(p))) \\
& \times \sum_{n=0}^{N-1} \exp \left(j \frac{2 \pi k(p-i)}{N}\right),
\end{aligned}
$$


where

$$
\exp \left(j \frac{2 \pi n(p-i)}{N}\right)= \begin{cases}N, & p=i \\ 0, & p \neq i\end{cases}
$$

Hence,

$$
\sigma_{\mathrm{CPE}}^{2}+\sigma_{\mathrm{ICI}}^{2}=E\left[\sum_{n=0}^{N-1}\left|g_{n}\right|^{2}\right]=\frac{1}{N^{2}}(N \times N)=1 .
$$

Using the DFT expression (13), $\sigma_{\mathrm{CPE}}^{2}$ can be derived as

$$
\begin{aligned}
E\left[\left|g_{0}\right|^{2}\right] & =E\left[\sum_{i} \sum_{p} \exp (j(\phi(i)-\phi(p)))\right] \\
& =\frac{1}{N^{2}} \sum_{i} \sum_{p} \exp \left(-2 \pi \beta|i-p| T_{\mathrm{s}}\right),
\end{aligned}
$$

where the last step is derived using the characteristic function (i.e., the Fourier transform of the probability density function) of the PN. Once $\sigma_{\mathrm{CPE}}^{2}$ is known, $\sigma_{\mathrm{ICI}}^{2}$ can be readily derived as

$$
E\left[\sum_{n \neq 0}\left|g_{n}\right|^{2}\right]=1-\frac{1}{N^{2}} \sum_{i} \sum_{p} \exp \left(-2 \pi \beta|i-p| T_{s}\right) .
$$

Equations (16)-(18) imply that the total energy of the PN is fixed and that reducing the subcarrier spacing reduces the CPE term and increases the ICI term.

Since $\mathbf{H}_{n}^{\dagger}=\left(\mathbf{H}_{n}^{H} \mathbf{H}_{n}\right)^{-1} \mathbf{H}_{n}^{H}$ and $E\left[\mathbf{w}_{n} \mathbf{w}_{n}^{H}\right]=\sigma_{w}^{2} \mathbf{I}_{M}$, the noise power (after the $\mathrm{ZF}$ decoder) is given as

$$
\begin{aligned}
E\left[\left\|\mathbf{H}_{n}^{\dagger} \mathbf{w}_{n}\right\|^{2}\right] & =\operatorname{Tr}\left\{E\left[\mathbf{H}_{n}^{\dagger} \mathbf{w}_{n} \mathbf{w}_{n}^{H}\left(\mathbf{H}_{n}^{\dagger}\right)^{H}\right]\right\} \\
& =\sigma_{w}^{2} \operatorname{Tr}\left\{E\left[\left(\mathbf{H}_{n}^{H} \mathbf{H}_{n}\right)^{-1}\right]\right\} .
\end{aligned}
$$

In the case of a Rayleigh fading multipath channel $\mathbf{H}_{n}^{H}$ and $M>K,\left(\mathbf{H}_{n}^{H} \mathbf{H}_{n}\right)^{-1}$ follows the inverse (noncentral) Wishart distribution, whose mean is $\mathbf{R}^{-1} /(M-K)$ [29]. Hence (19) boils down to

$$
E\left[\left\|\mathbf{H}_{n}^{\dagger} \mathbf{w}_{n}\right\|^{2}\right]=\frac{\sigma_{w}^{2}}{M-K} \operatorname{Tr}\left(\mathbf{R}^{-1}\right) .
$$

Note that the distribution of $\left(\mathbf{H}_{n}^{H} \mathbf{H}_{n}\right)^{-1}$ is unknown when $M=K$. Due to the singularity in (20), simulations for the case of $M=K$ do not converge. When $M<K$, the degree of freedom at the BS will be insufficient for detecting $K$ data streams. As a result, we only consider the case of $M>K$ in this paper.

Denoting the signal-to-noise ratio (SNR) as $\gamma_{0}=\sigma_{x}^{2} / \sigma_{w}^{2}$, the MSE at the $n$th subcarrier can be derived as follows:

$$
\xi_{n}=\frac{\gamma_{0} K\left(1-\left(1 / N^{2}\right) \sum_{i=0}^{N-1} \sum_{p=0}^{N-1} \exp \left(2 \pi \beta|i-p| T_{\mathrm{s}}\right)\right)+\left(\operatorname{Tr}\left(R^{-1}\right)\right) /(M-K)}{\left(\gamma_{0} K / N^{2}\right) \sum_{i=0}^{N-1} \sum_{p=0}^{N-1} \exp \left(2 \pi \beta|i-p| T_{\mathrm{s}}\right)} .
$$

As can be seen, $\xi_{n}$ is independent of the subcarrier index $n$; hence, the MSE averaged over all the subcarriers $\xi$ is also given by (21), that is, $\xi_{n}=\xi$. It can be concluded from (21) that the MSE performance degrades with increasing $\beta$ (the $3 \mathrm{~dB}$ bandwidth of the $\mathrm{PN}$ ) and/or with increasing number of users $K$ (for a given number of BS antennas $M$ ) and improves with increasing $M$ (for a given $K$ ).

For two users $(K=2)$ with a correlation of $\rho$ and power imbalance of $\eta_{1} / \eta_{2}$ (where $\eta_{1}$ and $\eta_{2}$ denote the MEGs of the two users' antennas, resp.),

$$
\operatorname{Tr}\left(\mathbf{R}^{-1}\right)=\left(\frac{1}{\eta_{1}}+\frac{1}{\eta_{2}}\right) \frac{1}{1-|\rho|^{2}} .
$$

In the absence of phase noises, the MSE reduces to

$$
\left.\xi\right|_{K=2}=\frac{\eta_{1}+\eta_{2}}{2 \gamma_{0}(M-2) \eta_{1} \eta_{2}\left(1-|\rho|^{2}\right)} .
$$

As can be seen from (23) the MSE performance degrades with increasing correlation and/or power imbalance and that the effects of correlation and power imbalance are separable (independent). Note that the two-user case is assumed in (22) and (23) in order to illustrate the effects of correlation and power imbalance explicitly, whereas (21) holds for any $K$ $(<M)$.

\section{Simulation}

Throughout the section, we make the following assumptions. There are 512 subcarriers including 32 scattered pilots. The remaining active subcarriers are loaded with QAM symbols. The multipath fading channel is a 4-tap Rayleigh fading channel, where the taps are at the $0,20,30$, and 60th time samples with equal average tap gain of $1 / 4$, and that the channel stays constant within 40 OFDM symbols after which an independent channel realization is drawn (i.e., block fading channel). In total, 100 channel realizations are generated. (The analysis in the previous section holds for Rayleigh fading channel with arbitrary number of channel taps; the 4-tap Rayleigh fading channel is used here as an example.) The CP length is set to 64 . The PNs from different users are independent yet follow the same (Wiener process) distribution with the same $3 \mathrm{~dB}$ PN bandwidth $\beta$. Without further specification, we assume $T_{\mathrm{s}}=10 \mathrm{~ns}$, corresponding to a bandwidth of $100 \mathrm{MHz}$, which can be realized using carrier aggregation in the current long-term evolution advanced (LTE-A) system and is decided as a typical bandwidth for $5 \mathrm{G}$ communications according to the 3GPP standardization [5]. For simplicity and in order to focus on the effects of PN, power imbalance, and correlation, we assume the channel has 


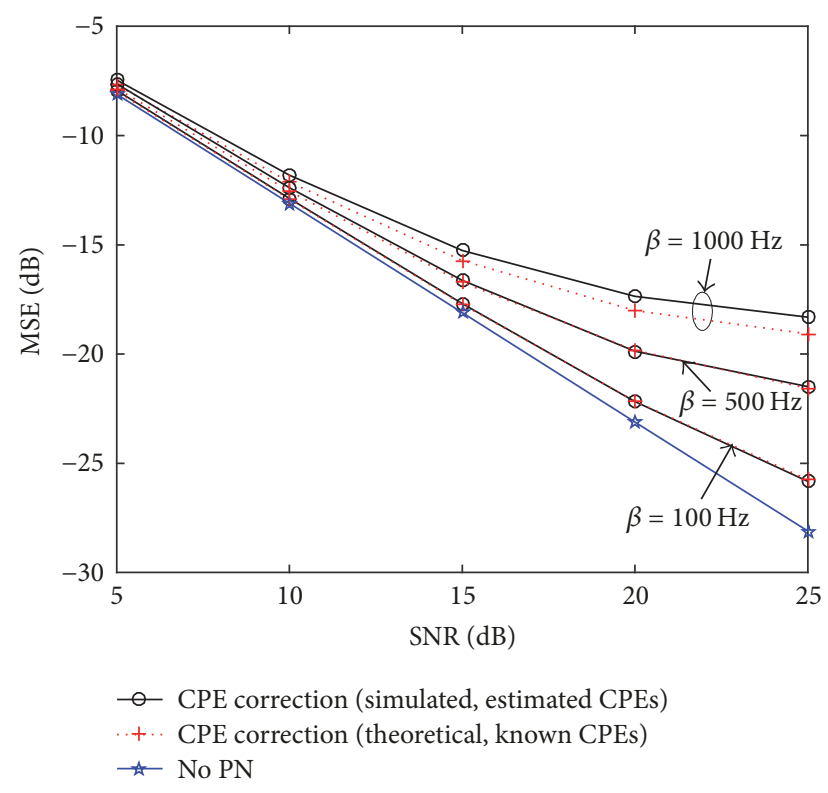

Figure 2: MSE performance of MU-MIMO-OFDM $(K=2, M=$ 4) with different $3 \mathrm{~dB} P N$ bandwidths in spatially white and powerbalanced MIMO channels.

been perfectly estimated in the preamble. The 32 scatter pilots are used for CPE estimation in the payload using (5).

Figure 2 shows the MSE performance of the MU-MIMOOFDM system with two users and four BS antennas in spatially white and power-balanced MIMO channels. As a reference, the ideal case (no PN) is also plotted in the same figure. As can be seen, with modest $\mathrm{PN}(\beta \leq 500 \mathrm{~Hz})$, the simulated MSE with CPE correction agrees well with that of the theoretical one (21). This implies that the CPE correction (cf. Section 2) can eliminate the CPEs of the multiple PNs. Note that as $\beta$ increases, the joint CPE estimation (5) becomes less accurate. As a result, it is also shown that the MSE performance with CPE correction is slightly worse than its theoretical counterpart as $\beta$ increases up to $1000 \mathrm{~Hz}$.

Figure 3 shows the MSE performance of the MU-MIMOOFDM system in spatially white and power-balanced MIMO channels as a function of subcarrier spacing $1 /\left(N T_{s}\right)$. Since the ICI effect reduces with increasing subcarrier spacing, the MSE performance improves with increasing subcarrier spacing. At $5 \mathrm{~dB} S N R$, the noises have more profound effect than the PNs do, whereas the PNs dominates at $25 \mathrm{~dB}$ SNR. Therefore, the improvement of the MSE performance (due to the increase of the subcarrier spacing) is more prominent at high SNR. Note that the channel length is assumed to be 61 time samples, whose absolute value (in seconds) decreases with increasing subcarrier spacing. For a fixed channel length (independent of the subcarrier spacing), it may not be a good idea to increase the subcarrier spacing unlimitedly in that a larger subcarrier spacing may necessitate a longer $\mathrm{CP}$ length (increased overhead) and multitap channel equalization (increased complexity).

Figure 4 shows the MSE performance of the PN corrupted MU-MIMO-OFDM system (with four users) in spatially white and power-balanced MIMO channels as a function of

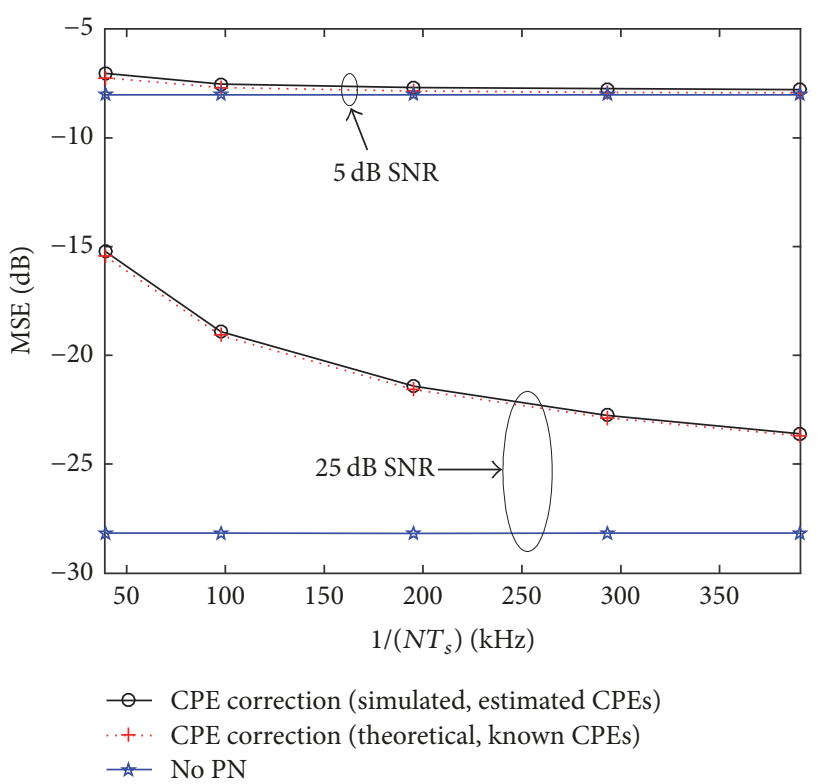

FIgURE 3: MSE performance of MU-MIMO-OFDM $(K=4$, $M=4, \beta=500 \mathrm{~Hz})$ as a function of subcarrier spacing $1 /\left(N T_{\mathrm{s}}\right)$ under different SNRs in spatially white and power-balanced MIMO channels.

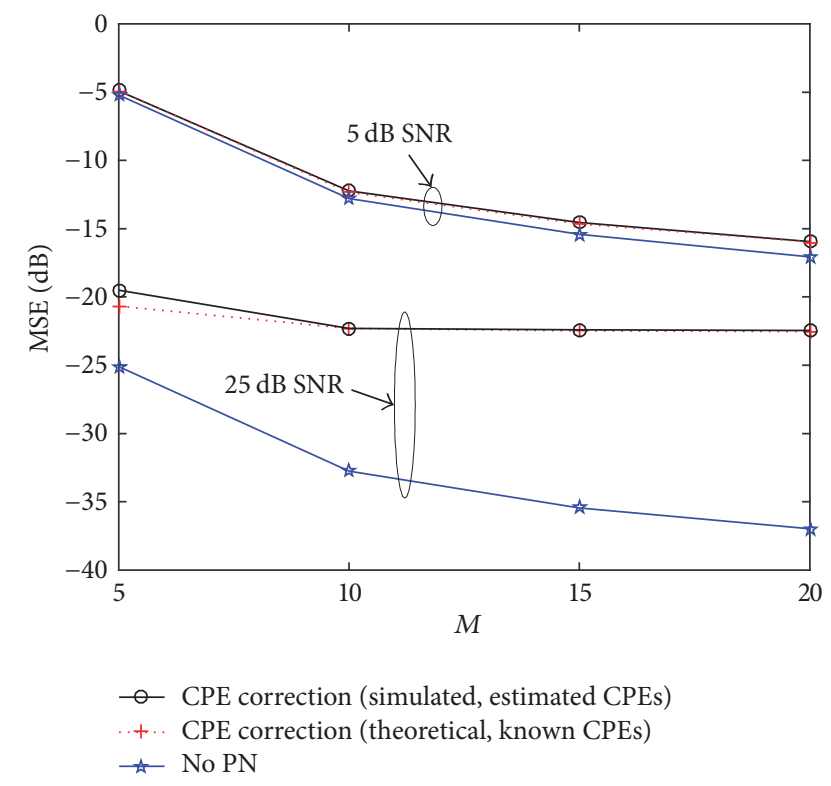

FIGURE 4: MSE performance of MU-MIMO-OFDM $(K=4, \beta=$ $500 \mathrm{~Hz}$ ) as a function of $M$ under different SNRs in spatially white and power-balanced MIMO channels.

number of BS antennas (under different SNRs). The $3 \mathrm{~dB}$ PN bandwidth is set to $500 \mathrm{~Hz}$. As references, the MSEs of the corresponding ideal cases (no PN) are also plotted in the same figure. As can be seen, the MSE performance improves as the number of BS antennas increases at low $(5 \mathrm{~dB})$ SNR. At high ( $25 \mathrm{~dB})$ SNR, however, the MSE performance improvement becomes insignificant beyond ten BS antennas. This is because that, given $K$, the diversity gain of the ZF 


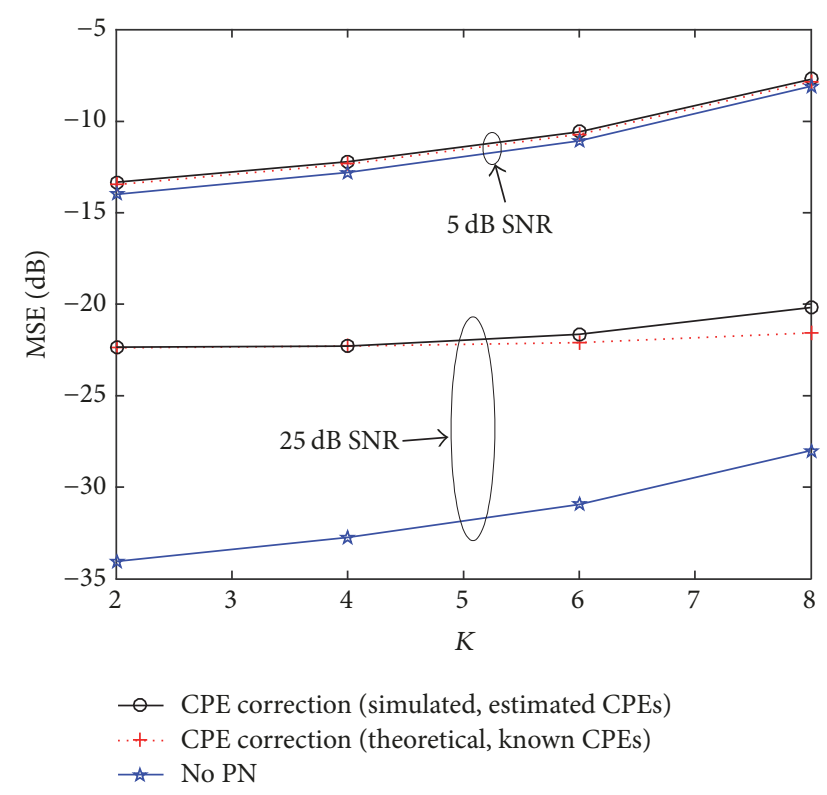

FIGURE 5: MSE performance of MU-MIMO-OFDM $(M=10, \beta=$ $500 \mathrm{~Hz}$ ) as a function of $K$ under different SNRs in spatially white and power-balanced MIMO channels.

decoder increases with increasing $M$; and the increasing diversity gain is more effective at low SNR.

Figure 5 shows the MSE performance of the PN corrupted MU-MIMO-OFDM system (with ten BS antennas) in spatially white and power-balanced MIMO channels as a function of number of users (under different SNRs). The $3 \mathrm{~dB}$ PN bandwidth is set to $500 \mathrm{~Hz}$. As references, the MSE of the corresponding ideal cases (no PN) are also plotted in the same figure. As can be seen, the MSE performance degrades as the number of users increases (for a given number of BS antennas). This is because that, given $M$, the diversity gain of the ZF decoder decreases with increasing $K$. Analogous to the results in Figure 4, the MSE dependence on the number of users is more obvious at low SNR than that at high SNR. Note that as the number of BS antennas $(M)$ decreases or as the number of users $(K)$ increases, the joint CPE estimation becomes less accurate, resulting in small discrepancies between the simulated and theoretical results in Figures 4 and 5.

To further illustrate the multiuser PN effects, we plot the MSE performances of MU-MIMO-OFDM systems with four BS antennas and two and three users, respectively, in Figure 6. The $3 \mathrm{~dB}$ PN bandwidth is set to $100 \mathrm{~Hz}$. As references, the MSEs of the corresponding ideal cases (no PN) are also plotted in the same figure. Given $M$, increasing $K$ decreases the degree of freedom at the BS and, therefore, degrades the MSE performance of the MU-MIMO-OFDM system. As can be seen from Figure 6, the MSE with the three users is higher than that with two users and that the theoretical results agree reasonably well with the simulated ones over the whole SNR range.

The above simulation results are for spatially white and power-balanced MIMO channels. The MSE performance of

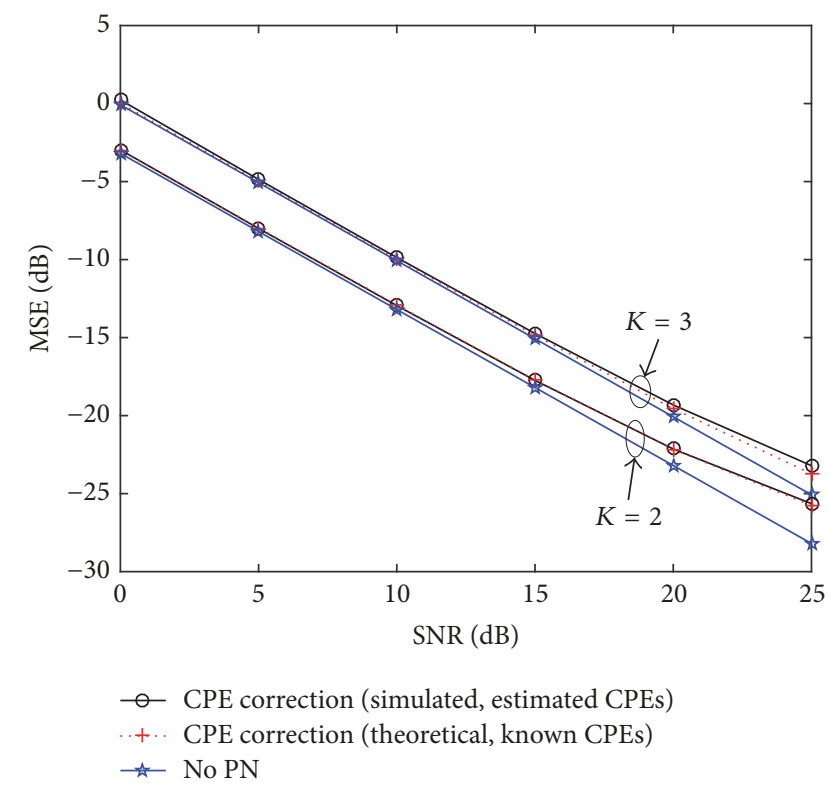

FIGURE 6: MSE performance of MU-MIMO-OFDM $(M=4, \beta=$ $100 \mathrm{~Hz}$ ) in spatially white and power-balanced MIMO channels.

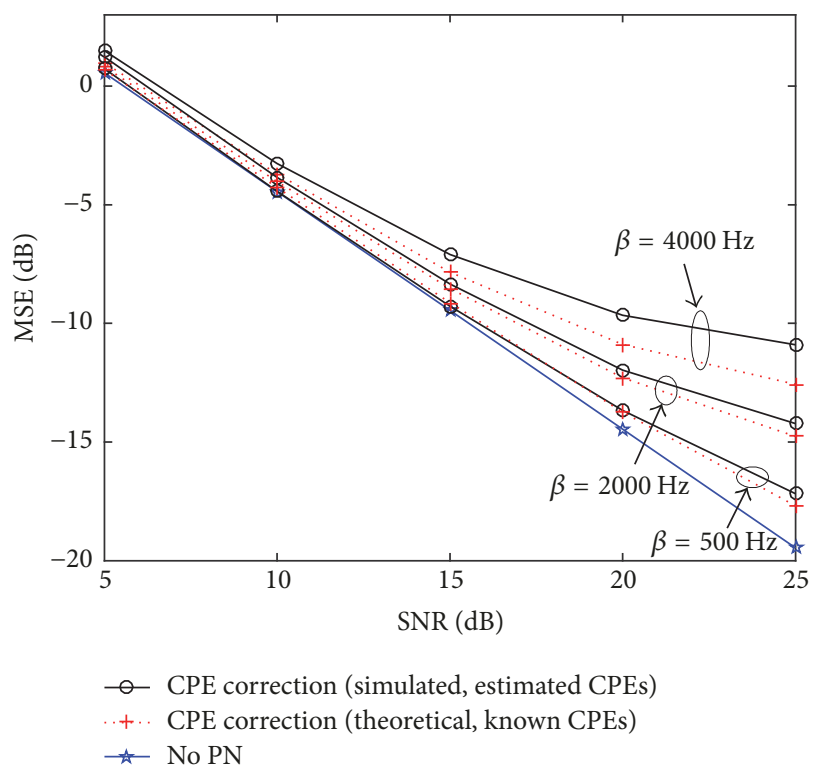

FIGURE 7: MSE performance of MU-MIMO-OFDM $(K=2, M=4)$ with different $3 \mathrm{~dB}$ PN bandwidths in the presence of 0.5 correlation and $10 \mathrm{~dB}$ power imbalance.

the MU-MIMO-OFDM system with two users and four BS antennas in the presence of 0.5 correlation and $10 \mathrm{~dB}$ power imbalance is shown in Figure 7. Comparing Figures 2 and 7, it can be seen that the power imbalance and the correlation significantly degrade the MSE performance of the MU-MIMO-OFDM system. Similar to Figure 2, as $\beta$ increases, the joint CPE estimation (5) becomes less accurate, and the difference between the simulated and theoretical MSE increases. 


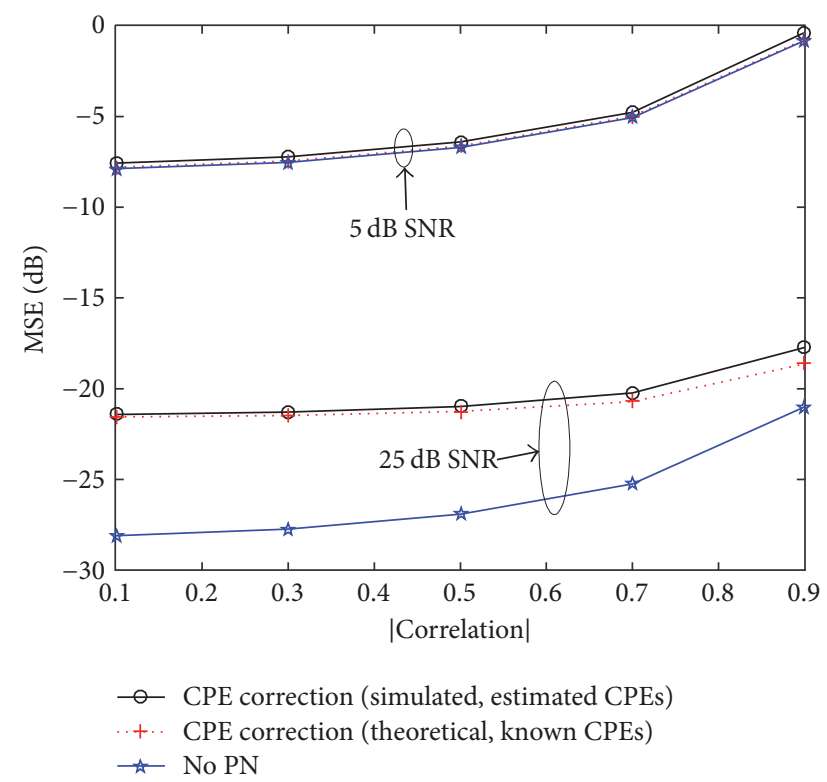

FIGURE 8: MSE performance of MU-MIMO-OFDM $(K=2, M=4$, $\beta=500 \mathrm{~Hz}$ ) as a function of user correlation in power-balanced MIMO channels.

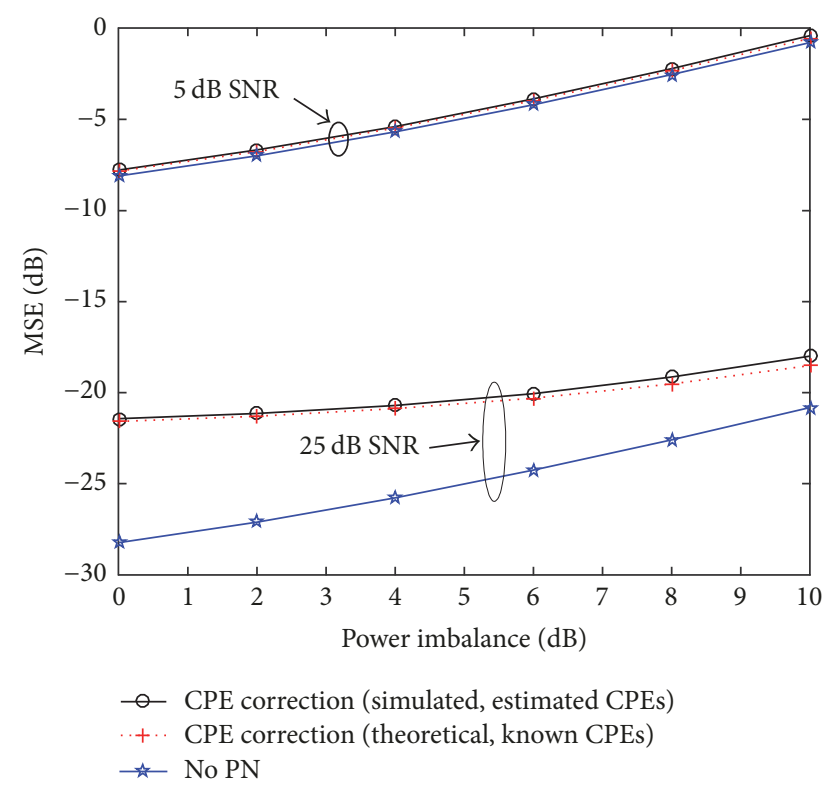

FIGURE 9: MSE performance of MU-MIMO-OFDM $(K=2, M=4$, $\beta=500 \mathrm{~Hz}$ ) as a function of power imbalance in spatially white MIMO channels.

It is shown from (22) that the effects of correlation and power imbalance are separable. Thus, we show their effects on the MU-MIMO-OFDM system with two users and four BS antennas in Figures 8 and 9, respectively. As can be seen, the MSE performance degrades with increasing correlation and/or power imbalance and that the theoretical MSE expression (21) can well predict the simulated MSE. The small discrepancy at high correlation (power imbalance) is

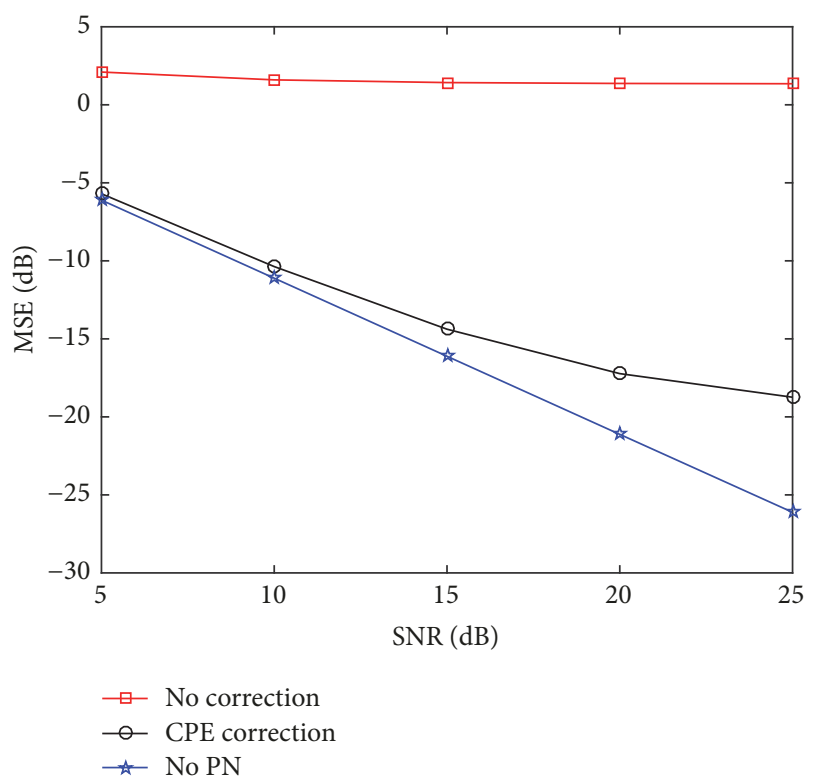

FIgURE 10: MSE performance of MU-MIMO-OFDM $(K=2, M=$ $4, \beta=500 \mathrm{~Hz}$ ) with PNs at both users and the BS in the presence of 0.3 correlation and $3 \mathrm{~dB}$ power imbalance.

due to the fact that the estimated CPEs become less accurate at high correlation (power imbalance) values.

So far, we have been focusing on the effect of multiuser PNs, while omitting the PN at the BS. As shown in Section 2, the $\mathrm{CPE}$ of the PN at the BS can be corrected together with the multiuser CPEs using the CPE correction method. Figure 10 shows the MSE performance of the MU-MIMOOFDM system with two users and four BS antennas in the presence of 0.3 correlation and $3 \mathrm{~dB}$ power imbalance, and PNs (with $\beta=500 \mathrm{~Hz}$ ) at users and the BS. As can be seen, the PNs of the users and the BS can be greatly mitigated by using the $\mathrm{CPE}$ correction method.

\section{Conclusions}

In this work, we studied the phase noise (PN) effect on the uplink multiuser MIMO-OFDM system. It was assumed that each user is equipped with a single antenna along with a freerunning oscillator, whereas the base station (BS) has multiple antennas and use zero-forcing for multiuser detection. An analytical mean square error (MSE) expression was derived for the case where the common phase errors (CPEs) can be perfectly estimated. The analytical MSE expression can take into account of correlations and power imbalances between different users. It was shown that the MSE performance improves with increasing number of BS antennas $(M)$ yet degrades with increasing power imbalance, correlation, number of users $(K)$, and/or $3 \mathrm{~dB}$ PN bandwidth and that the MSE dependence on $M$ or $K$ is more obvious at low SNR. In the special case of two users, the correlation and power imbalance effects are separable (independent) and can be shown explicitly in the analytical MSE expression. The analytical results were verified by simulations. 


\section{Conflicts of Interest}

The authors declare that they have no conflicts of interest.

\section{Acknowledgments}

The research leading to these results received funding from the European Commission H2020 Programme under Grant Agreement no. 671650 (5G PPP mmMAGIC project).

\section{References}

[1] J. A. C. Bingham, "Multicarrier modulation for data transmission: an idea whose time has come," IEEE Communications Magazine, vol. 28, no. 5, pp. 5-14, 1990.

[2] R. He, B. Ai, G. L. Stüber, G. Wang, and Z. Zhong, "Geometrical based modeling for millimeter wave MIMO mobile-to-mobile channels," IEEE Trans. Veh. Technol, vol. 67, 2018.

[3] K. Guan, B. Ai, A. Fricke et al., "Excess Propagation Loss of Semi-Closed Obstacles for Inter/Intra-Device Communications in the Millimeter-Wave Range," Journal of Infrared, Millimeter, and Terahertz Waves, vol. 37, no. 7, pp. 676-690, 2016.

[4] R. He, Q. Li, B. Ai et al., "A Kernel-power-density based algorithm for channel multipath components clustering," IEEE Trans. Wireless Commun, vol. 16, no. 11, pp. 7138-7151, 2017.

[5] http://www.3gpp.org/.

[6] G. J. Foschini, "Layered space-time architecture for wireless communication in a fading environment when using multielement antennas," Bell Labs Technical Journal, vol. 1, no. 2, pp. 41-59, 1996.

[7] W. Fan, I. Carton, P. Kyosti et al., "A Step Toward 5G in 2020: Low-cost OTA performance evaluation of massive MIMO base stations.," IEEE Antennas and Propagation Magazine, vol. 59, no. 1, pp. 38-47, 2017.

[8] B. Ai, K. Guan, R. He et al., "On Indoor Millimeter Wave Massive MIMO Channels: Measurement and Simulation," IEEE J. Sel. Areas Commun, vol. 35, no. 7, pp. 1678-1690, 2017.

[9] M. Barazzetta, D. Micheli, L. Bastianelli et al., "A Comparison between Different Reception Diversity Schemes of a 4G-LTE Base Station in Reverberation Chamber: A Deployment in a Live Cellular Network," IEEE Transactions on Electromagnetic Compatibility, vol. 59, no. 6, pp. 2029-2037, 2017.

[10] R. Recanatini, F. Moglie, and V. M. Primiani, "Performance and immunity evaluation of complete wlan systems in a large reverberation chamber," IEEE Transactions on Electromagnetic Compatibility, vol. 55, no. 5, pp. 806-815, 2013.

[11] T. C. W. Schenk, X.-J. Tao, P. F. M. Smulders, and E. R. Fledderus, "On the influence of phase noise induced ICI in MIMO OFDM systems," IEEE Communications Letters, vol. 9, no. 8, pp. 682684, 2005.

[12] J. Li and W. Wu, "Performance of MIMO-OFDM Systems with Phase Noise at Transmit and Receive Antennas," in Proceedings of the 2011 7th International Conference on Wireless Communications, Networking and Mobile Computing (WiCOM), pp. 1-4, Wuhan, China, September 2011.

[13] S. Bittner, E. Zimmermann, and G. Fettweis, "Exploiting phase noise properties in the design of MIMO-OFDM receivers," in Proceedings of the IEEE Wireless Communications and Networking Conference, WCNC 2008, pp. 940-945, usa, April 2008.

[14] R. Corvaja and A. G. Armada, "SINR degradation in MIMOOFDM systems with channel estimation errors and partial phase noise compensation," IEEE Transactions on Communications, vol. 58, no. 8, pp. 2199-2203, 2010.

[15] R. Hamila, Ö. Özdemir, and N. Al-Dhahir, "Beamforming OFDM Performance Under Joint Phase Noise and I/Q Imbalance," IEEE Transactions on Vehicular Technology, vol. 65, no. 5, pp. 2978-2989, 2016.

[16] O. H. Salim, W. Xiang, and A. A. Nasir, Joint data detection and phase noise mitigation for light field video transmission in MIMO-OFDM systems, https://arxiv.org/abs/1602.02834.

[17] X. Chen, H. Wang, W. Fan et al., "Phase Noise Effect on MIMOOFDM Systems with Common and Independent Oscillators," Wireless Communications and Mobile Computing, vol. 2017, pp. 1-12, 2017.

[18] P. Xu, Y. Xiao, S. Zhou, and M. Zhao, "ICI analysis of MIMOOFDM systems with independent phase noise at both transmit and receive antennas," in Proceedings of the 5th International Conference on Wireless Communications, Networking and Mobile Computing (WiCOM '09), Beijing, China, September 2009.

[19] R. Krishnan, M. R. Khanzadi, N. Krishnan et al., On the impact of oscillator phase noise on the uplink performance in a massive MIMO-OFDM system, https://arxiv.org/abs/1405.0669.

[20] S. Wu and Y. Bar-Ness, "Multiple phase noise correction for OFDM/SDMA," in Proceedings of the GLOBECOM '03. IEEE Global Telecommunications Conference, pp. 1311-1315, San Francisco, CA, USA.

[21] A. Pitarokoilis, E. Björnson, and E. G. Larsson, "Performance of the Massive MIMO Uplink with OFDM and Phase Noise," IEEE Communications Letters, vol. 20, no. 8, pp. 1595-1598, 2016.

[22] A. Puglielli, G. Lacaille, A. M. Niknejad, G. Wright, B. Nikolic, and E. Alon, "Phase noise scaling and tracking in OFDM multiuser beamforming arrays," in Proceedings of the 2016 IEEE International Conference on Communications (ICC '16), 6, 1 pages, Kuala Lumpur, Malaysia, May 2016.

[23] X. Chen, "OFDM Based Multi-Node Transmission in the Presence of Phase Noises for Small Cell Backhaul," IEEE Communications Letters, vol. 21, no. 5, pp. 1207-1210, 2017.

[24] W. Xu, S. A. Zekavat, and H. Tong, "A novel spatially correlated multiuser MIMO channel modeling: Impact of surface roughness," IEEE Transactions on Antennas and Propagation, vol. 57, no. 8, pp. 2429-2438, 2009.

[25] A. Adhikary, E. Al Safadi, M. K. Samimi et al., "Joint spatial division and multiplexing for mm-Wave channels," IEEE Journal on Selected Areas in Communications, vol. 32, no. 6, pp. 1239-1255, 2014.

[26] X. Gao, O. Edfors, F. Rusek, and F. Tufvesson, "Linear precoding performance in measured very-large MIMO channels," in Proceedings of the IEEE Vehicular Technology Conference (VTC Fall '11), pp. 1-5, IEEE, San Francisco, Calif, USA, September 2011.

[27] X. Chen, S. Zhang, and A. Zhang, "On MIMO-UFMC in the Presence of Phase Noise and Antenna Mutual Coupling," Radio Science, vol. 52, no. 11, pp. 1386-1394, 2017.

[28] X. Chen and S. Zhang, "Multiplexing efficiency for MIMO antenna-channel impairment characterisation in realistic multipath environments," IET Microwaves, Antennas \& Propagation, vol. 11, no. 4, pp. 524-528, 2017.

[29] P. Graczyk, G. Letac, and H. Massam, "The complex Wishart distribution and the symmetric group," The Annals of Statistics, vol. 31, no. 1, pp. 287-309, 2003. 


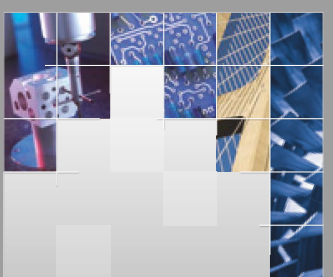

\section{Enfincering}
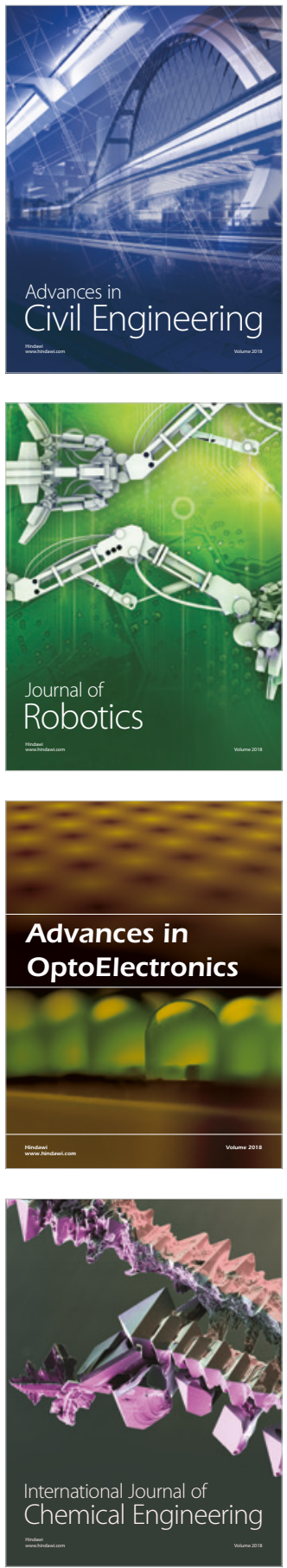

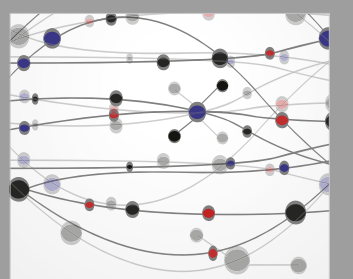

\section{Rotating \\ Machinery}

The Scientific World Journal

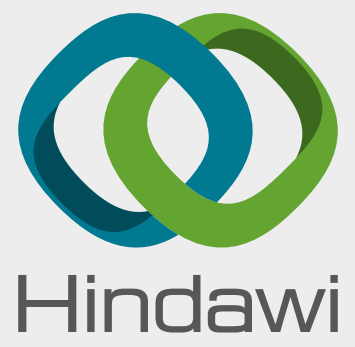

Submit your manuscripts at

www.hindawi.com
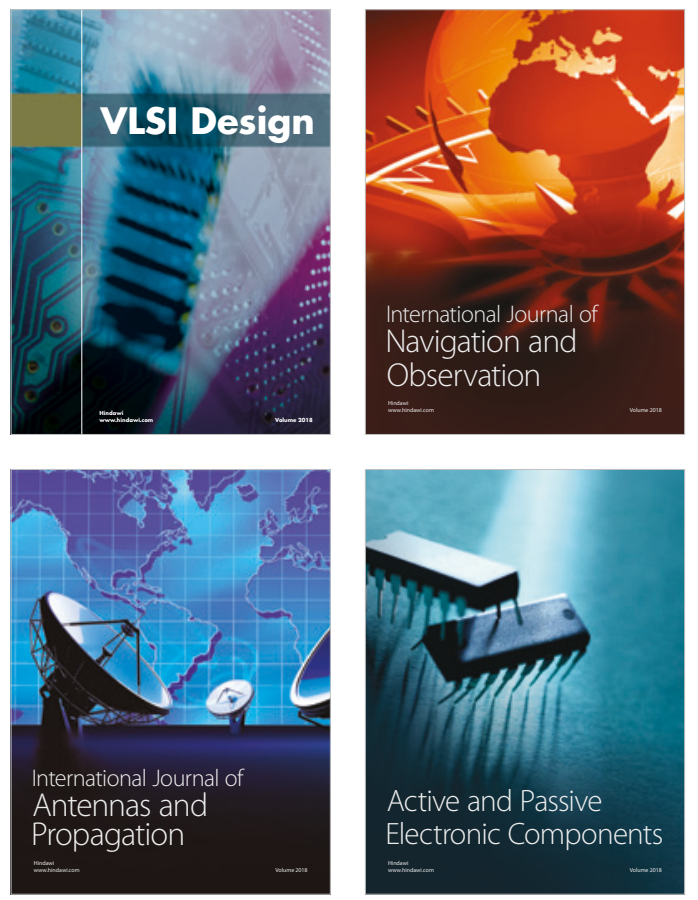
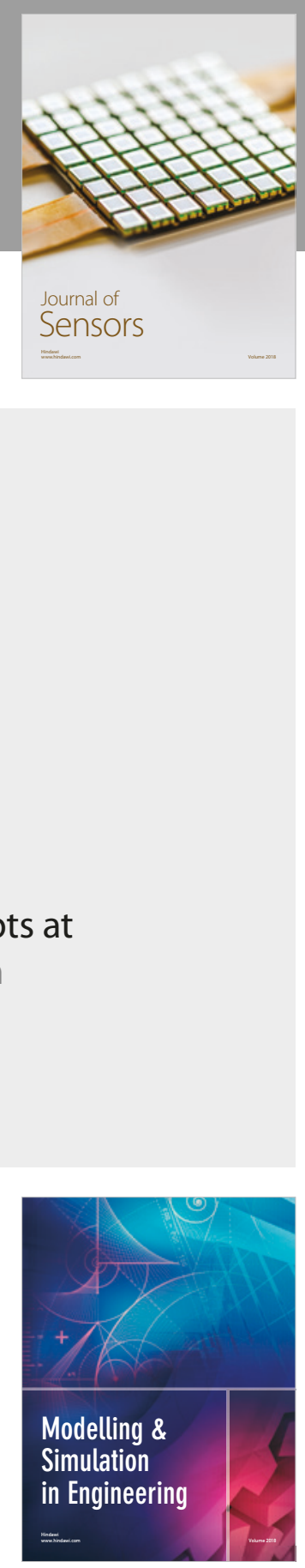

\section{Advances \\ Multimedia}
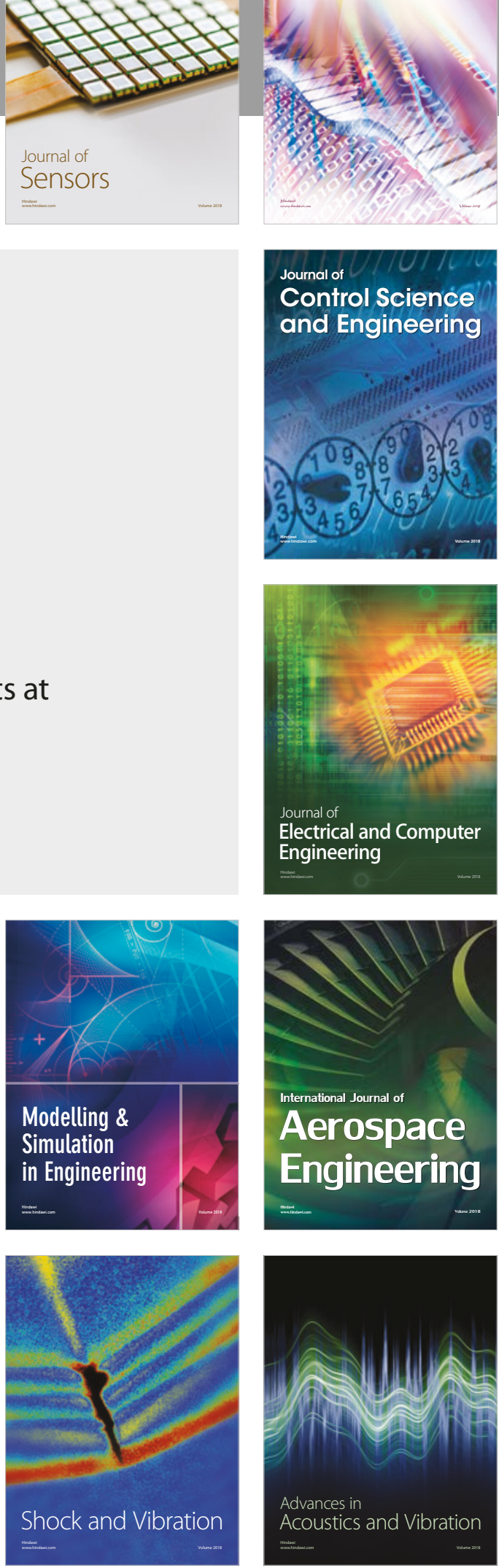